\title{
Effect of plyometric training on jumping performance and agility in adolescent badminton players
}

\author{
Tarik OZMEN 1, Mert AYDOGMUS ${ }^{2}$ \\ ${ }^{1}$ Department of Physiotherapy and Rehabilitation, Faculty of Health Sciences, Karabuk University, Karabuk, Turkey. \\ ${ }^{2}$ Hasan Dogan School of Physical Education and Sports, Karabuk University, Karabuk, Turkey. \\ Address correspondence to T. Ozmen, e-mail: tarikozmen@karabuk.edu.tr
}

\begin{abstract}
Badminton is one of the most popular racquet sports in the world. The agility and vertical jump are important motor skills to hit a shuttlecock in badminton game. The purpose of this study was to investigate effect of plyometric training on vertical jump and agility in adolescent badminton players. Twenty adolescent (age, $12.5 \pm 0.2$ years) badminton players were randomly divided into two groups as plyometric group (PG) and control group (CG). All participants were tested to assess agility and vertical jump before (pre-test) and after 6-weeks training period (post-test). Agility performance was assessed with Illinois Agility Test. Vertical jump height was measured with squat jump test using a contact mat. The PG performed plyometric training twice a week, for 6 weeks. There was a significant difference between pre and post-test for squat jump test in both PG $(26 \%)(p=0.00)$ and CG $(10 \%)(p=0.016)$. However, squat jump height significantly increased in PG compared with CG $(p=$ $0.024)$. The agility significantly improved in PG $(6 \%)(p=0.01)$, but not CG $(2 \%)(p=0.294)$. Our results show that a six week plyometric training improved agility and vertical jump in adolescent badminton players. Badminton game requires fast changes in direction, vertical jumps, forward lunges around the court. Badminton coaches should take place plyometric exercises in training program.
\end{abstract}

Keywords: Children, plyometrics, squat jump.

\section{INTRODUCTION}

Plyometrics are training method including explosive-type exercises used by athletes in all of sports (5). Plyometric exercises consist of two phases; a rapid stretching of a muscle (eccentric action) and a rapid shortening (concentric action) (11). The stored elastic energy within the muscle during stretching is used to produce more force following concentric phase. Plyometric exercises may facilitate peripheral and central neural adaptations that increase joint proprioception and kinesthetic awareness. A rapid stretch and shortening activity may lead to sensitivity of the muscle spindles and desensitization of the Golgi tendon organs during eccentric loading (27). Previous studies have been reported that plyometric training was effective in improving muscle strength, vertical jump, speed, and agility in both children and adults $(17,18,22,24,30)$. Meylan \& Malatesta (17) found significant improvement in 10-m sprint time by $22.1 \%$ and agility test time by $29.6 \%$ and significant increase in jump height following an 8week plyometric program in adolescent soccer players. Rubley et al. (24) observed that plyometric training improved vertical jump after 14 weeks in female adolescent soccer players. Miller et al. (18) showed that a six week plyometric training program improved two agility test performance in young subjects.

Badminton is one of the most popular racquet sports in the world (14). The game of badminton includes intermittent rallies of high intensity on court $(14,15)$. The badminton match requires fast changes in direction, vertical jumps, forward lunges, and different postural positions (15). Tiwari et al. (29) observed that agility was related to physical performance ( $\mathrm{r}=0.83$ ) during a badminton match. The shuttlecock's atypical and surprising flight trajectory requires considerable skill for hitting it (1). To our knowledge, the agility and vertical jump are important motor skills to hit a shuttlecock at different positions around the court.

To our knowledge, there are limited number of studies investigating to the effects of plyometric training on athletic performance in badminton 
players $(8,9)$. Heang et al. (9) showed that a six week plyometric training improved agility performance in young badminton players. The aim of this study was to investigate effects of plyometric training on vertical jump and agility in adolescent badminton players.

\section{MATERIALS \& METHODS}

Twenty adolescent badminton players (9 boys, 11 girls; age $=12.5 \pm 0.2$ years) participated in this study. All participants had prior badminton experience of at least 2 year. None of the subjects had lower-extremity injuries and surgery within 6 months prior to the pre-tests, any cardiovascular and metabolic disease. This study was approved by the University Institutional Review Committee for the ethical use of human subjects.

All participants were tested to evaluate agility and vertical jump before (pre-test) and after 6-weeks (post-test). Participants were randomly divided into two groups of plyometric (PG) and control (CG). The PG performed plyometric training twice a week for 6 weeks in addition to the routine training. Both groups maintained twice a week badminton training throughout the study period. The CG did not participate in any plyometric exercises at the time of the study.

Agility performance was assessed with Illinois Agility Test (23). The test area with a length of $10 \mathrm{~m}$ and a width of $5 \mathrm{~m}$ was set up with four cones on the basketball court. Another four cones were placed down in the center with spaced $3.3 \mathrm{~m}$ apart. The photocells were located to start and finish lines. The subjects were instructed to run as quickly as possible around the course. The best result of the two trials with five minutes recovery between trials was recorded.

Vertical jump height was measured with squat jump test using a contact mat (Swift Performance Equipment, Lismore, NSW, Australia). The squat jump test is one of the most reliable and valid field tests to evaluate of explosive power of the lower extremities measured by contact mat (16). Each participant jumped with $90^{\circ}$ knee flexion, barefoot, and their hands on hips on the mat. The participants were instructed to keep $90^{\circ}$ knee flexion position for four seconds before jumping for maximum height. The best score of two jumping effort was recorded.

The participants performed plyometric exercises twice a week for a 6-week period. The plyometric training program included progressive exercises similar to the training protocol of Miller et al. (18) was used (Table 1).

Table 1. Plyometric training program.

\begin{tabular}{|c|c|c|c|}
\hline Week & $\begin{array}{l}\text { Training } \\
\text { Volume }\end{array}$ & Exercises & $\begin{array}{l}\text { Sets } x \\
\text { Reps }\end{array}$ \\
\hline \multirow[t]{3}{*}{1} & \multirow[t]{3}{*}{90} & Side to side ankle hops & $2 \times 15$ \\
\hline & & Standing jump and reach & $2 \times 15$ \\
\hline & & Front cone hops & $5 \times 6$ \\
\hline \multirow[t]{4}{*}{2} & \multirow[t]{4}{*}{120} & Side to side ankle hops & $2 \times 15$ \\
\hline & & Standing long jump & $5 \times 6$ \\
\hline & & Lateral jump over barrier & $2 \times 15$ \\
\hline & & Double leg hops & $5 \times 6$ \\
\hline \multirow[t]{4}{*}{3} & \multirow[t]{4}{*}{120} & Side to side ankle hops & $2 \times 15$ \\
\hline & & Standing long jump & $5 \times 6$ \\
\hline & & Lateral jump over barrier & $2 \times 15$ \\
\hline & & Double leg hops & $5 \times 6$ \\
\hline \multirow[t]{5}{*}{4} & \multirow[t]{5}{*}{134} & Lateral jump over barrier & $2 \times 15$ \\
\hline & & Single leg bounding & $4 \times 7$ \\
\hline & & Lateral jump single leg & $4 \times 6$ \\
\hline & & Diagonal cone hops & $4 \times 8$ \\
\hline & & Cone hops with 180 degree turn & $4 \times 5$ \\
\hline \multirow[t]{5}{*}{5} & \multirow[t]{5}{*}{134} & Lateral jump over barrier & $2 \times 15$ \\
\hline & & Single leg bounding & $4 \times 7$ \\
\hline & & Lateral jump single leg & $4 \times 6$ \\
\hline & & Diagonal cone hops & $4 \times 8$ \\
\hline & & Cone hops with 180 degree turn & $4 \times 5$ \\
\hline \multirow[t]{5}{*}{6} & \multirow[t]{5}{*}{134} & Diagonal cone hops & $2 \times 15$ \\
\hline & & Hexagon drill & $2 \times 12$ \\
\hline & & Cone hops with 180 degree turn & $4 \times 6$ \\
\hline & & Double leg hops & $4 \times 8$ \\
\hline & & Lateral jump single leg & $4 \times 6$ \\
\hline
\end{tabular}

\section{Statistical Analysis}

All analyses were completed using SPSS (Version 16.0, SPSS Inc, Chicago, IL). The normality of distribution for dependent variables was confirmed with the Shapiro-Wilk Test. Results of the pre- and post-tests were compared using the Pairedsample $t$ test within each group. The differences between pre- and post-tests of the groups analysed with Independent t-tests (2-tailed). Statistical significance was set at $p \leq .05$. Cohen $d$ effect size and effect-size confidence intervals were calculated. Effect sizes were interpreted as small $(0.2-.49)$, medium (0.50-0.79), and large $(\geq 0.8)(18)$. 


\section{RESULTS}

Descriptive characteristics of participants are shown in Table 2. There were no significant differences between PG and CG based on age, height, and weight $(p>.05)$. The effects of the training (with $95 \%$ confidence limits) on performance of the groups are presented in Table 3. Paired-sample t-test indicated that there was a significant difference between pre and post-test for Squat Jump Test in PG $(26 \%$, medium effect size, $p=0.00)$ and CG $(10 \%$, small effect size, $p=0.016)$. However, Independent $t-$ test showed that squat jump height significantly increased in PG compared with CG (medium effect size, $\mathrm{p}=0.02$ ). Agility significantly improved in $\mathrm{PG}$ $(6 \%$, small effect size, $\mathrm{p}=0.01)$, but not CG $(2 \%, \mathrm{p}=$ $0.294)$.

Table 2. Descriptive characteristics of groups.

\begin{tabular}{lccccc}
\hline \multirow{2}{*}{ Variable } & \multicolumn{2}{l}{ PG $(n=10 ; 4$ boys, 6 girls $)$} & \multicolumn{2}{c}{ CG $(n=10 ;(5$ boys, 5 girls $)$} & \\
\cline { 2 - 6 } & Mean & SD & Mean & SD & $\mathrm{p}$ \\
\hline Age (yrs) & 12.0 & 0.0 & 12.1 & 0.3 & 0.33 \\
Height $(\mathrm{cm})$ & 150.3 & 6.2 & 150.9 & 6.6 & 0.83 \\
Weight $(\mathrm{kg})$ & 38.4 & 10.7 & 44.7 & 15.2 & 0.30 \\
\hline
\end{tabular}

Abbreviations: PG, plyometric group; CG, control group

Table 3. Training effects (with 95\% confidence limits) for the performance variables of groups.

\begin{tabular}{llccccc}
\hline Test & Group & Pre-Test & Post-Test & Performance Change (\%) & Effect Size & p \\
\hline Squat Jump (cm) & PG & $15.98 \pm 2.76$ & $20.18 \pm 3.63^{\mathrm{a}}$ & +26 & 0.54 & $0.024^{\mathrm{b}}$ \\
& CG & $17.10 \pm 4.77$ & $18.94 \pm 3.95^{\mathrm{a}}$ & +10 & 0.20 & \\
Agility (s) & PG & $20.04 \pm 1.21$ & $18.82 \pm 1.23^{\mathrm{a}}$ & +6 & 0.44 & $0.008^{\mathrm{b}}$ \\
& CG & $21.28 \pm 1.70$ & $21.74 \pm 1.63$ & -2 & 0.13 & \\
\hline
\end{tabular}

Abbreviations: PG, plyometric group; CG, control group.

a Significant difference from pre-test $(p<.05) .{ }^{\mathrm{b}}$ Significant difference from control group $(p<.05)$.

\section{DISCUSSION}

Plyometric training involves explosive concentric contractions of the muscles via the stretch reflex and produce high stress on muscles and bones. Plyometric training is considered unsafe for children, and a prerequisite for participation previous experience in strength training (11). On the other hand, some studies demonstrated that plyometric exercises improved whole body bone mineral content and stimulated bone formation in children $(12,31$, 33). The present training program did not lead to any injuries or symptoms of discomfort. Previous authors documented that well designed plyometric training programs were effective for improving sprint, agility and vertical jump abilities in children $(3,17,19,20)$. The present study showed that a six week plyometric training significantly increased squat jump height by
$26 \%$ in adolescent badminton players. The improvement in jump height may be a result of the neural adaptations because the training period is shorter than eight weeks. These adaptations involve an increase in $\alpha$-motoneuron firing frequency and recruitment. Plyometric training may improve voluntary activation of muscles by inducing specific adaptations at the supraspinal and/or spinal level depending on the contraction mode (2). The period of plyometric training was shorter than those of other studies in the present study. However, to our knowledge, this improvement could be explained that the participants in this study had no experience of plyometric training. On the other hand, the squat jump height increased by $10 \%$ from pre-test to posttest in the CG. It is possible that badminton training contributed to vertical jump by improving explosive strength in the lower extremities. The result of the 
current study is in accordance with the results of previous studies in trained and untrained children. Kotzamanidis (13) reported that squat jump height (34\%) increased following a ten week plyometric training in nonathletic boys. They suggested that improvement in jump height could be due to the rate of force development, power, and stiffness enhancement. Ramirez-Campillo et al. (20) showed that squat jump height increased by $18 \%$ after 7 weeks of plyometric training in nontrained adolescent males. Also, they suggested that training adaptations were associated with hardness of the training surface used during plyometric training. Chelly et al. (4) reported that a 10-week plyometric training led to increase in the squat jump height (14\%) in adolescent track athletes. The authors observed total leg and thigh muscle volumes remained unchanged after plyometric training. They suggested that the gains in jump height were due to various neuromuscular adaptations involving the stretch reflex and the storage of elastic energy. The athletes performed three times per week plyometric training. In the control group, was observed low increase by $5 \%$ compared with our control group (10\%). Also, the athletes had mean experience of the training by 3.4 years higher than those of our participants. Rubley et al. (24) found that plyometric training increased vertical jump height by $8.3 \%$ from pre-test to 7 weeks in adolescent soccer players. In this study, one per week plyometric exercises with low impact was used compared with frequency of our training program. Meylan \& Malatesta (17) observed significant increase by $7.9 \%$ in counter movement jump height, but not squat jump height following an eight week plyometric program in children soccer players. They explained that squat jump height did not improve because squat jump involving minimal stretchshortening cycle of the muscle compared with counter movement jump was not stimulated during training. There is only one study investigating the effect of plyometric training on vertical jump in adolescent badminton players. Fröhlich et al. (8) showed that the plyometric training twice a week for eight weeks resulted in an increase by $13.7 \%$ in squat jump height. But, there was no control group.

The discrepancies between our results and those of the previous studies could be explained by the differences in the intensity, frequency and period of the plyometric training and previous fitness level of the children. Also, few authors showed that the gains of the plyometric training were affected by training surface $(10,20,26)$. In the present study, plyometric training was performed in gymnasium floor on hard surface, but not sand or grass.

Another result of the present study was that a six week plyometric training improved by $6 \%$ agility time for Illinois Agility Test. Agility has been described as the ability to maintain a controlled body position and fast change of directions (32). To our knowledge, plyometric exercises involve explosive lower extremity movements and fast muscle contractions. Therefore, our plyometric training program might be contributed to agility development. Heang et al. (9) observed that a six week plyometric training significantly improved agility performance in both plyometric (7\%) and control group $(2.5 \%)$ in young badminton players. The training intensity and exercises was similar to our training program. But, they performed plyometric training one session per week. They suggested that co-curriculum badminton training program contributed to development of agility of the control group (9). Meylan \& Malatesta (17) found that an 8-week plyometric program significantly improved agility test time $(29.6 \%)$ in children soccer players. Thomas et al. (28) compared the effects of two plyometric training methods on power and agility in youth soccer players. After six weeks, they found significant decrease in agility times. Miller et al. (18) reported that agility times improved $2.93 \%$ for Illinois Agility Test after two sessions per week a six week plyometric training program in young subjects. The training intensity and plyometric exercises was similar to our training program. Ramirez-Campillo et al. (21) documented that Illinois Agility Test times significantly decreased by $3.5 \%$ following 7 weeks of plyometric training in adolescent soccer players.

One limitation of the present study is that no physiological measurements (e.g. electromyography) were taken to observe neural adaptations induced by the plyometric training. Another limitation of the study was short training period compared with other studies. However, our training program improved both vertical jump and agility. The effects of plyometric training could vary depending on factors such as gender, age, training level, type of sport activity, familiarity with plyometric training, training duration, and training intensity (7).

The findings of this study showed that a six week plyometric training improved agility and vertical jump in adolescent badminton players. Badminton is one of the most common sports among children in many countries. The badminton match requires fast changes in direction, jumps, forward 
lunges around the court. Consequently, the agility and vertical jump are important motor skills to win competition in badminton game. Coaches should incorporate in badminton training program plyometric exercises.

\section{ACKNOWLEDGEMENT}

This study was supported by Scientific Research Project Unit of Karabuk University (KBÜ-BAP-15/1KP-036). The author thanks to Semih Uzer due to support of the implementation of the training program.

\section{REFERENCES}

1. Alam F, Chowdhury H, Theppadungporn C, Subic A, Masud $\mathrm{M}$, Khan K. Aerodynamic properties of badminton shuttlecock. International Journal of Mechanical and Materials Engineering, 2009; 4 (3): 266-72.

2. Behrens M, Mau-Moeller A, Mueller K, Heise S, Gube M, Beuster N, Bruhn S. Plyometric training improves voluntary activation and strength during isometric, concentric and eccentric contractions. Journal of Science and Medicine in Sport, 2016; 19(2): 170-176.

3. Chelly MS, Hermassi S, Aouadi R, Shephard RJ. Effects of 8week in-season plyometric training on upper and lower limb performance of elite adolescent handball players. Journal of Strength and Conditioning Research, 2014; 28(5): 1401-1410.

4. Chelly MS, Hermassi S, Shephard RJ. Effects of in-season short-term plyometric training program on sprint and jump performance of young male track athletes. Journal of Strength and Conditioning Research, 2015; 29(8): 2128-2136.

5. Chu DA. Jumping into plyometrics. Champaign, IL: Human Kinetics; 1998.

6. Cohen J. A power primer. Psychological Bulletin, 1992; 112(1) 155 .

7. De Villarreal ES S, Kellis E, Kraemer WJ, Izquierdo M. Determining variables of plyometric training for improving vertical jump height performance: a meta-analysis. Journal of Strength and Conditioning Research, 2009; 23(2): 495-506.

8. Fröhlich M, Felder H, Reuter M. Training effects of plyometric training on jump parameters in D-and D/C-squad badminton players. Journal of Sports Research, 2014; 1(2): 22-33.

9. Heang LJ, Hoe WE, Quin CK, Yin LH. Effect of plyometric training on the agility of students enrolled in required college badminton programme. International Journal of Applied Sports Science, 2012; 24(1): 18-24.

10. Impellizzeri FM, Rampinini E, Castagna C, Martino F, Fiorini $\mathrm{S}$, Wisloff $\mathrm{U}$. Effect of plyometric training on sand versus grass on muscle soreness and jumping and sprinting ability in soccer players. British Journal of Sports Medicine, 2008; 42(1): 42-46.

11. Johnson BA, Salzberg CL, Stevenson DA. A systematic review: Plyometric training programs for young children. Journal of Strength and Conditioning Research, 2011; 25(9): 2623-2633.

12. Kish K, Mezil Y, Ward WE, Klentrou P, Falk B. Effects of plyometric exercise session on markers of bone turnover in boys and young men. European Journal of Applied Physiology, 2015; 115(10): 2115-2124.
13. Kotzamanidis C. Effect of plyometric training on running performance and vertical jumping in prepubertal boys. Journal of Strength and Conditioning Research, 2006; 20(2): 441-445.

14. Lees A. Science and the major racket sports: a review. Journal of Sports Sciences, 2003; 21(9): 707-732.

15. Manrique DC, Gonzalez-Badillo JJ. Analysis of the characteristics of competitive badminton. British Journal of Sports Medicine, 2003; 37(1): 62-66.

16. Markovic G, Dizdar D, Jukic I, Cardinale M. Reliability and factorial validity of squat and countermovement jump tests. Journal of Strength and Conditioning Research, 2004; 18(3): 551-555.

17. Meylan C, Malatesta D. Effects of in-season plyometric training within soccer practice on explosive actions of young players. Journal of Strength and Conditioning Research, 2009; 23(9): 2605-2613.

18. Miller MG, Herniman JJ, Ricard MD, Cheatham CC, Michael TJ. The effects of a 6-week plyometric training program on agility. Journal of Sports Science and Medicine, 2006; 5(3): 459465.

19. Mulcahy RL, Crowther RG. The effects of an 8 week supplemented plyometric exercise training program on leg power, agility and speed in adolescent netball players. Journal of Australian Strength and Conditioning, 2013; 21(3): 31-6.

20. Ramirez-Campillo R, Andrade DC, Izquierdo M. Effects of plyometric training volume and training surface on explosive strength. Journal of Strength and Conditioning Research, 2013; 27(10): 2714-2722.

21. Ramirez-Campillo R, Meylan C, Alvarez C, Henriquez-Olguin C, Martinez C, Canas-Jamett R, Izquierdo M. Effects of inseason low-volume high-intensity plyometric training on explosive actions and endurance of young soccer players. Journal of Strength and Conditioning Research, 2014; 28(5): 1335-1342.

22. Ramirez-Campillo R, Vergara-Pedreros $M$, Henriquez-Olguin C, Martinez-Salazar C, Alvarez C, Nakamura FY, Izquierdo M. Effects of plyometric training on maximal-intensity exercise and endurance in male and female soccer players. Journal of Sports Sciences, 2016; 34(8): 687-693.

23. Raya MA, Gailey RS, Gaunaurd IA, Jayne DM, Campbell SM, Gagne E, Tucker C. Comparison of three agility tests with male servicemembers: Edgren Side Step Test, T-Test, and Illinois Agility Test. Journal of Rehabilitation Research and Development, 2013; 50(7): 951-60.

24. Rubley MD, Haase AC, Holcomb WR, Girouard TJ, Tandy RD. The effect of plyometric training on power and kicking distance in female adolescent soccer players. Journal of Strength and Conditioning Research, 2011; 25(1): 129-134.

25. Saunders PU, Telford RD, Pyne DB, Peltola EM, Cunningham RB, Gore CJ, Hawley JA. Short-term plyometric training improves running economy in highly trained middle and long distance runners. Journal of Strength and Conditioning Research, 2006; 20(4): 947-954.

26. Stemm JD, Jacobson BH. Comparison of land-and aquaticbased plyometric training on vertical jump performance. Journal of Strength and Conditioning Research, 2007; 21(2): 568-571.

27. Swanik KA, Lephart SM, Swanik CB, Lephart SP, Stone DA, Fu $\mathrm{FH}$. The effects of shoulder plyometric training on 
proprioception and selected muscle performance characteristics. Journal of Shoulder Elbow Surgery, 2002; 11(6) 579-586.

28. Thomas K, French D, Hayes PR. The effect of two plyometric training techniques on muscular power and agility in youth soccer players. Journal of Strength and Conditioning Research, 2009; 23(1): 332-335.

29. Tiwari LM, Rai V, Srinet S. Relationship of selected motor fitness components with the performance of badminton player. Asian Journal of Physical Education and Computer Science in Sports, 2011; 5(1): 88-91.

30. Vaczi M, Tollar J, Meszler B, Juhasz I, Karsai I. Short-term high intensity plyometric training program improves strength, power and agility in male soccer players. Journal of Human Kinetics, 2013; 36(1): 17-26.

31. Witzke KA, Snow CM. Effects of polymetric jump training on bone mass in adolescent girls. Medicine and Science in Sports and Exercise, 2000; 32(6): 1051-1057.

32. Young W, Farrow D. A Review of Agility: Practical Applications for Strength and Conditioning. Strength and Conditioning Journal, 2006; 28(5): 24-29.

33. Zribi A, Zouch M, Chaari H, Bouajina E, Ben NH, Zaouali M, Tabka Z. Short-term lower-body plyometric training improves whole-body BMC, bone metabolic markers, and physical fitness in early pubertal male basketball players. Pediatric Exercise Science, 2014; 26(1): 22-32. 